\title{
Athermal bladder neck dissection during robot-assisted radical prostatectomy
}

\author{
Fabrizio Dal Moro \\ Department of Surgical, Oncological and Gastroenterological Sciences, University of Padova, Padova, Italy
}

\section{ABSTRACT}

Introduction: With improved understanding of the precise anatomy, surgical techniques during robot-assisted radical prostatectomy (RARP) have been refined, with the aim of improving functional outcomes without compromising oncological adequacy and results. Nevertheless, postoperative urinary incontinence remains a frustrating side-effect. Anatomically, bladder neck (BN) serves as an internal sphincter. The longitudinal fibres of BN may be identified and isolated with a meticulous dissection at the prostato-vesical junction, contributing to earlier return of urinary continence. The purpose of this video is to show an anatomical athermal dissection of BN during RARP.

Materials and Methods: After incision of endopelvic fascia and anterior defatting, the morphology of prostate not only laterally, but also at the level of bladder-prostatic junction is well visualized.

With an athermal dissection of the plane between prostate and bladder we can minimize the traumatic effects on the longitudinal fibres of BN. A cold section of the preserved BN permits the complete preservation of integrity of this sphincteric structure.

Results: With this technique we preserve the longitudinal fibres of BN, allowing the sparing of the sphincteric mechanism of BN. The finding of a difficult athermal dissection of these plans may make you suspect the presence of an infiltration, suggesting to sacrifice BN in order to avoid a positive surgical margin. In our series no increase of PSM has been recorded using this technique.

Conclusions: This surgical technique preserving the natural BN mechanism appears to improve urinary continence, allowing at the same time an easy identification of a neoplastic infiltration.

\section{ARTICLE INFO}

Available at: www.brazjurol.com.br/videos/may_june_2014/Dal_Moro_433_434video.htm

Int Braz J Urol. 2014; 40 (Video \#8): 433-4

Submitted for publication:

January 10, 2014

Accepted after revision:

May 15, 2014
Correspondence address:

Fabrizio Dal Moro, MD Assistant Professor

Department of Surgical, Oncological and Gastroenterological Sciences

University of Padova

Via Giustiniani 2

Padova, 35126, Italy

Fax: +3 904 9821-8757

E-mail: fabrizio.dalmoro@unipd.it 


\section{EDITORIAL COMMENT}

In this very elegant video depiction by Del Moro et al., robotic assisted laparoscopic prostatectomy offers great benefits in improving the surgical visualization and manual dexterity to performing key steps of this surgical procedure. The surgeon(s) of the present video beautifully depict how an anatomically meticulous bladder neck preserving approach can be conducted whereby optimizing early and long-term urinary continence. Although such an approach can be technically conducted for most surgically managed prostate cancer cases, it is pivotal to adapt the surgical technique to the specific location of the tumor burden i.e. if significant high volume and phenotypically aggressive (Gleason grade $7(4+3)$ or more) is situated at the base of prostate based on pre-operative TRUS biopsy results with or without correlative endorectal MRI of the prostate findings, I would caution surgeons to perform such an extensive bladder neck sparing approach and if so, diligent use of intra-operative frozen section and/or adoption of this surgical technique based on intra-operative findings would be prudent. In summary, like many facets of surgical oncology, an operative procedure must be personalized to the anatomical and tumor characteristics of the individual patient.

Philippe E. Spiess, MD Associate Member, Department of Genitourinary Oncology, Moffitt Cancer Center Video Section Editor, International Brazilian Journal of Urology 